\title{
THE OPTICAL AND ELECTRICAL PROPERTIES OF ZnO/Ag/ZnO FILMS ON FLEXIBLE SUBSTRATE*
}

\author{
Xiaojing Yu, Dongyan Zhang \\ MOE Key Laboratory for Non-equilibrium Synthesis and Modulation of Condensed Matter, School of \\ Science, Xi'an Jiaotong University, Shaanxi 710049, People's Republic of China
}

Department of Mechanical Engineering, The University of Tokushima, 2-1 Minamijosanjima-cho, Tokushima 770-8506,Japan.yxj8867@yahoo.cn youngcool@gmail.com

\author{
Pangpang Wang Ri-ichi Murakami ${ }^{\mathrm{a}}$
}

Department of Mechanical Engineering, The University of Tokushima, 2-1 Minamijosanjima-cho, Tokushima 770-8506,Japan.wangpp@cicee.tokushima-u.ac.jp murakami@me.tokushima-u.ac.jp

\section{Bingjun Ding, Xiaoping Song}

MOE Key Laboratory for Non-equilibrium Synthesis and Modulation of Condensed Matter, School of Science, Xi'an Jiaotong University, Shaanxi 710049, People's Republic of China bjding@mail.xjtu.edu.cn, xpsong@mail.xjtu.edu.cn

\begin{abstract}
The deposition of $\mathrm{ZnO} / \mathrm{Ag} / \mathrm{ZnO}$ film on polyethylene terephthalate (PET) substrate was fabricated by DC magnetron sputtering method. The thicknesses of $\mathrm{ZnO}$ layers were $30 \mathrm{~nm}$ and $\mathrm{Ag}$ films' thicknesses were changed from $1 \mathrm{~nm}$ to $6 \mathrm{~nm}$ by controlled the sputtering time. This kind of film can be used as transparent conductive oxide (TCO) materials. The electrical and optical properties of composite layers were determined by Ag films. The optimum sputtering time of Ag thin films was found to be $20 \mathrm{~s}$ for the high optical transmittance with good electrical conductivity. The $\mathrm{ZnO} / \mathrm{Ag}(20 \mathrm{~s}) / \mathrm{ZnO}$ layer, which has high optical transmittance of $73 \%$ at $550 \mathrm{~nm}$, shows sheet resistance as low as $6.7 \mathrm{ohm} / \mathrm{sq}$.. These multilayer transparent films had low electrical resistance as the widely used transparent conductive oxide electrodes. SEM, XRD, the UV-Vis-NIR and Hall Effect measurement system were used to characterize properties of fabricated films. The reasons for the change of transmittance and resistance will also be interpreted.
\end{abstract}

Keywords: Transparent conductive oxide (TCO); Magnetron Sputtering; $\mathrm{ZnO} / \mathrm{Ag} / \mathrm{ZnO}$.

\section{Introduction}

Nowadays, with the development of electronic production, optoelectronic technology has been paid great deal of attentions by scientists. It is widely known that the optical transparency of semiconductor material requires their band gaps be larger than $3 \mathrm{eV}$, which is difficult for electron carrier transfer into conduction band. As the result, it is generally believed that high optical transparency is incompatible with high electronical conduction. ${ }^{1}$ However, the situation is changed since transparent conductive oxide (TCO) 
films have been extensively studied. TCO materials hold on the ability that combining the good electrical conduction with well transparency of visible light. ${ }^{2}$ This kind of material is wildly exploited into optical-electronics, flat panel displays, solar cells etc. because of its outstanding performance of transparency and conductivity. ${ }^{3-5}$ The fabrication and properties of metal oxides such as $\mathrm{In}_{2} \mathrm{O}_{3}, \mathrm{SnO}_{2}$ and $\mathrm{ZnO}$ have been comprehensively studied. In general, indium tin oxide (ITO) thin films are widely used as transparent conductive electrodes. Nevertheless, it is the high cost and rarity of indium that hamper the further development of ITO material. $\mathrm{ZnO}$ was noticed by much more eyes because it is nonpoisonous, low cost, and abundance. $\mathrm{ZnO}$ is an typical n-type semiconductor with a wide band gap of $3.37 \mathrm{eV}{ }^{6}$ The $\mathrm{ZnO}$, which works as a dielectric material with a high refractive index, can serve as good material for TCO as got united with low index metal, $(\mathrm{Ag}, \mathrm{Cu})$. Therefore, it has been considered as a promising candidate for application of TCO material.

Recent developments in the TCO material field have generated substantial interests for fabricating TCO films on flexible substrates, ${ }^{7}$ because the flexible substrate can overcome the inevitable disadvantage of glass-like substrate, such as brittle, heavy weight and nondeformable. Polyethylene terephthalate (PET) and polyethylene napthalate (PEN) are widely studied because they are light weight, dimensionally stable, chemically resistant, and hold on good ability of flexible nature.

In this study, the $\mathrm{ZnO} / \mathrm{Ag} / \mathrm{ZnO}$ sandwich structured films have been fabricated using magnetron sputtering. This method has lots of merits, such as wide area deposition, high deposition rate, and simple equipment and operating means. The crystal structure and morphology of fabricated film are revealed by X-ray diffraction (XRD) and scanning electron microscope (SEM) respectively. Optical transmittance spectrum and Hall Effect measurement (works as resistance test instrument), are used to exhibit the optical and electronic conductivity properties.

\section{Experiments}

The $\mathrm{ZnO}$ and $\mathrm{ZnO} / \mathrm{Ag} / \mathrm{ZnO}$ thin films were deposited on PET substrates with using a zinc $(99.99 \%$ purity, $300 \mathrm{~mm} \times 62 \mathrm{~mm} \times 5 \mathrm{~mm})$ and metal Ag targets $(99.99 \%$ purity, $300 \mathrm{~mm} \times 62 \mathrm{~mm} \times 5 \mathrm{~mm}$ ) by a magnetron sputter deposition system(NACHI, SP-1530-1), which is equipped with DC power suppliers. The Zn target and Ag target were settled opposite, that in order to reduce the interaction and influence between each other. The sputtering was performed with a target has $45^{\circ}$ degree angle with substrate. The PET substrate was cleaned by acetone and ethanol solution in ultrasonic cleaning apparatus, dried subsequently and pasted on to substrate holders smoothly and flatly before deposition. The $\mathrm{Zn}$ target was sputtered in the Oxygen and Argon atmosphere, with $3 \mathrm{sccm}$ and $50 \mathrm{sccm}$ flow-rate respectively. During the sputtering, the $\mathrm{Zn}$ and Oxygen react with each other to form $\mathrm{ZnO}$ particles and then the $\mathrm{ZnO}$ particles grew on the substrate to make up a film. The Ag target was sputtered in the Argon atmosphere, with about $50 \mathrm{sccm}$, that prevent the reaction with other gases. In order to discuss the influence of Ag layers, the thickness of the $\mathrm{ZnO}$ layer was controlled on $30 \mathrm{~nm}$ and those 
layers of $\mathrm{Ag}$ were varied between $1 \mathrm{~nm}$ to $6 \mathrm{~nm}$ approximately. The sputtering time and thickness of samples are listed in Table I.

Table I Thickness of each layer for samples

\begin{tabular}{|c|c|c|c|c|c|c|}
\hline \multirow{2}{*}{ Sample } & \multicolumn{3}{|c|}{ Sputtering time } & \multicolumn{3}{c|}{ Thickness of layer } \\
\cline { 2 - 7 } & $\begin{array}{c}\text { Bottom ayer } \\
\mathrm{ZnO}(\mathrm{min})\end{array}$ & $\begin{array}{c}\mathrm{Ag} \\
(\mathrm{s})\end{array}$ & $\begin{array}{c}\text { Top layer } \\
\mathrm{ZnO}(\mathrm{min})\end{array}$ & $\begin{array}{c}\text { Bottom layer } \\
\mathrm{ZnO}(\mathrm{nm})\end{array}$ & $\begin{array}{c}\mathrm{Ag} \\
(\mathrm{nm})\end{array}$ & $\begin{array}{c}\text { Top layer } \\
\mathrm{ZnO}(\mathrm{nm})\end{array}$ \\
\hline ZnO/Ag5/ZnO & 60 & 5 & 60 & 30 & 1 & 30 \\
\hline ZnO/Ag10/ZnO & 60 & 10 & 60 & 30 & 2 & 30 \\
\hline ZnO/Ag15/ZnO & 60 & 15 & 60 & 30 & 3 & 30 \\
\hline ZnO/Ag20/ZnO & 60 & 20 & 60 & 30 & 4 & 30 \\
\hline ZnO/Ag25/ZnO & 60 & 25 & 60 & 30 & 5 & 30 \\
\hline ZnO/Ag30/ZnO & 60 & 30 & 60 & 30 & 6 & 30 \\
\hline
\end{tabular}

The optical transmittance spectrum was measured by UV-Vis-NIR spectrometer (JASCO, V570) in the wavelength range of $300 \mathrm{~nm}$ to $800 \mathrm{~nm}$. The Sheet resistance of electrical properties was measured by a Hall Effect measurement system, which is following the Van der Pauw method (4-point probe method). The morphologic properties of the Ag layer were observed by using field emission scanning electron microscopy (FESEM, Hitachi S-4700). The crystalline structures of the $\mathrm{ZnO}$ films with embedded Ag were studied by X-ray diffraction (XRD, Rigaku-RINT 2500) with $\mathrm{Cu} \mathrm{K \alpha}$ radiation operating at $30 \mathrm{kV}$ and $100 \mathrm{~mA}$, while the data were collected with a step size of $0.02^{\circ}$ and scanning speed of $4^{\circ}$ per min.

\section{Results and discussion}

Fig. 1 presents the XRD patterns of deposited $\mathrm{ZnO} / \mathrm{Ag} / \mathrm{ZnO}$ multilayer. There are a strong (002) orientation peak for $\mathrm{ZnO}$ and (111) orientation peak of Silver, that indicating the polycrystalline nature of the films. The other two peaks might be contributed by the amorphism of PET substrate, as it can be also seen in the XRD pattern of bare PET substrate without any layers (Fig.1 b).

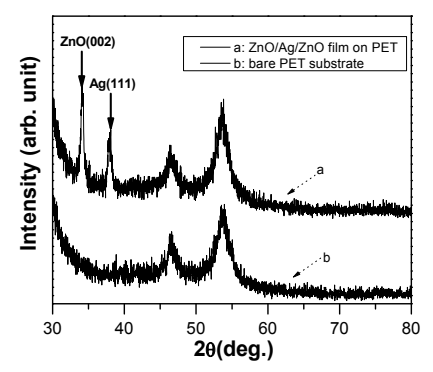

Fig 1. XRD patterns of $\mathrm{ZnO} / \mathrm{Ag} / \mathrm{ZnO}$ film on PET (a) and bare PET substrate (b)

Fig. 2 shows the relationships between transmittance and wavelength of each sample with different thicknesses of $\mathrm{Ag}$ layer, while two $\mathrm{ZnO}$ layers are fixed on $30 \mathrm{~nm}$. The 
pure $\mathrm{ZnO}$ layer, which sputtering on PET substrates, shows a persistently increased transmittance from $300 \mathrm{~nm}$ to $800 \mathrm{~nm}$. The transmittances of every fabricated degenerate film all have a peak around 470 600 nm and the transmittances are higher than $60 \%$ through the visible light wavelength range. In the long wavelength region, there are transmittance decreases that accompanied with the increment of the silver layer thickness. With the increasing of silver, the transmittance's decreasing speed is escalated. The reduced transmittance was due to the light absorption which related with the imaginary part of the complex dielectric function of $\mathrm{Ag} .{ }^{8}$ In the visible light wavelength region, there is an improvement of transmittance at the original stage of Ag film deposition when sputtering time of $\mathrm{Ag}$ thickness is increased from $5 \mathrm{~s}$ to $20 \mathrm{~s}$. While, with further increasing of Ag layers' sputtering time to $30 \mathrm{~s}$, a decrement of transmittance could be observed. When the light wavelength was fixed on $540 \mathrm{~nm}$, the relationship between changing transmittance of the fabricated thin films and Ag sputtering time is shown by Fig.3. Obviously, the $\mathrm{ZnO} / \mathrm{Ag}(20 \mathrm{~s}) / \mathrm{ZnO}$ has higher transmittance than the others. The morphology of the $4 \mathrm{~nm} \mathrm{Ag}$ thin film, which showed the best transparent property, is showed the SEM images (Fig.4). The exhibited Ag film has a discontinued island nanostructure. It is the unclosed packed structure that provides a well place to allow light pass though.

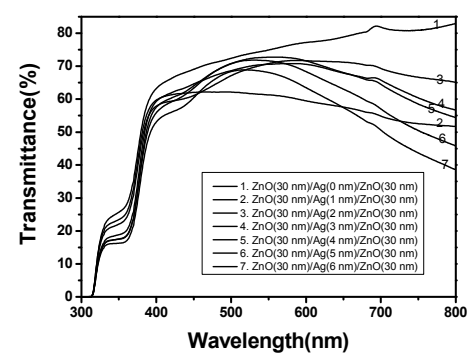

Fig. 2 The optical transmittance spectra of $\mathrm{ZnO} / \mathrm{Ag} / \mathrm{ZnO}$ films

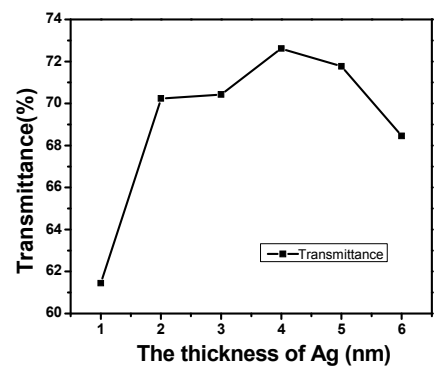

Fig. 3 The curve of transmittance changing as a function Ag sputtering time

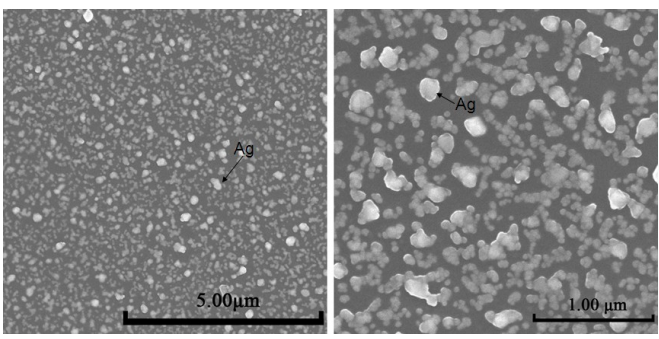

Fig. 4 SEM images for $\mathrm{ZnO} / \mathrm{Ag}(20 \mathrm{~s}) / \mathrm{Ag}$ film. Left image is $\times 10.0 \mathrm{k}$. Right image is $\times 30.0 \mathrm{k}$

The sheet resistances of the fabricated layers were measured by the four-point probe Hall Effect system. The sheet resistance of the pure $\mathrm{ZnO}$ layer could not be measured 
since the resistance exceeds the measurable range of measure system. The sheet resistance is decreased as the sputtering time of $\mathrm{Ag}$ layer is increased (As Fig.5 shown). Evidently, the decrease of sheet resistances is due to the increase of Ag layers thickness. The sheet resistance of the multilayer system with the time of Ag sputtering equal to $5 \mathrm{~s}$ is $125.41 \mathrm{ohm} / \mathrm{sq}$. It is shown that even small amount of Ag particles could help the film to get ability of electrical conduction. This phenomenon could be explained as followed: Commonly, the electrons in the metal oxides (e.g. $\mathrm{ZnO}$ ) have small effective masses. When the Ag particle is sputtered, the electrons change to high-density distribution. As the result, the conduction ability of fabricated object is improved. The sheet resistance has a sharp decrease from $125.41 \mathrm{ohm} / \mathrm{sq}$. to $21.45 \mathrm{ohm} / \mathrm{sq}$. when the Ag sputtering time was increased to $10 \mathrm{~s}$, but as content of Ag particle was increasing, the decrease of sheet resistance was leveling off. When the Ag sputtering time add to $30 \mathrm{~s}$, the sheet resistance shows the lowest value at $3.89 \mathrm{ohm} / \mathrm{sq}$.

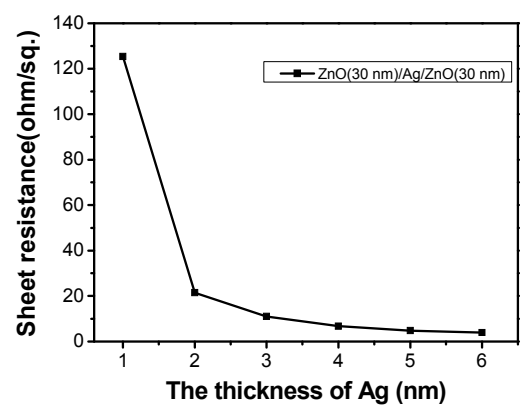

Fig. 5 The Dependence of sheet resistance on the $\mathrm{Ag}$ thickness for $\mathrm{ZnO} / \mathrm{Ag} / \mathrm{ZnO}$ films on PET substrates

\section{Conclusion}

Briefly, different multilayers of $\mathrm{ZnO} / \mathrm{Ag} / \mathrm{ZnO}$ on PET substrates have been fabricated by $\mathrm{DC}$ magnetron sputtering. Within the increase of $\mathrm{Ag}$ metal layer thickness, the electrical properties of the films had received a good reform. However, considered to balance of the electrical and optical properties, in this paper, the $\mathrm{ZnO} / \mathrm{Ag} / \mathrm{ZnO}$ layer, which sputtering silver by $20 \mathrm{~s}$ shows the highest transmittance and low sheet resistance. These results prove that the sandwich structured $\mathrm{ZnO} / \mathrm{Ag} / \mathrm{ZnO}$ film, which sputtered on flexible substrate (PET), also has acceptable properties of combining the high transmittance and low resistance. So this kind of TCO material can be probably used in flexible electronic field.

\section{Acknowledgment}

This study was supported by the Double Degree Program (DDP) of The University of Tokushima and Xi'an Jiaotong University that advocated by Ministry of Education, Culture, Sports, Science \& Technology (MEXT), Japan. 


\section{References}

1. H. Hosono Thin Solid Films 515 (2007) 6000-6014

2. H. Han, D. Adams, J. W. Mayer, and T. L. Alford, J. Appl. Phys. 98, (2005) 083705

3. A. Chipman, Nature (London) 449, (2007)131

4. Y.R. Park, D. Jung, Y.S. Kim, Jpn, J. Appl. Phys. 47 (2008) 516.

5. C.Q. Zhang, J.P. Tu, Y.F. Yuan, X.H. Huang, X.T. Chen, F. Mao, J. Electrochem. Soc. 154 (2007) A65.

6. M. Matsumura, Renato, P. Camata, Thin Solid Films 476 (2005) 317.

7. K. Sivaramakrishnan, N. D. Theodore, J. F. Moulder, T. L. Alford, J. Appl. Phys. 106, (2009)063510

8. D.Y.Zhang, P.P. Wang, R. Murakami, X.P.Song Appl. Phys. Lett. 96, (2010)233114 